\title{
Dark-field Transmission Electron Microscopy Imaging Technique to Visualize the Local Structure of Two-dimensional Material; Graphene
}

\author{
Min Young $\mathrm{Na}^{1,2}$, Seung-Mo Lee ${ }^{3}$, Do Hyang Kim², Hye Jung Chang ${ }^{1,4, *}$ \\ ${ }^{1}$ Advanced Analysis Center, Korea Institute of Science and Technology, Seoul 136-791, Korea \\ ${ }^{2}$ Center for Non-Crystalline Materials, Yonsei University, Seoul 120-749, Korea \\ ${ }^{3}$ Nano-Convergence Mechanical Systems Research Division, Korea Institute of Machinery \& Materials, Daejeon 305-343, Korea \\ ${ }^{4}$ Department of Nanomaterials Science and Technology, Korea University of Science and Technology, Daejeon 305-350, Korea
}

*Correspondence to:

Chang $\mathrm{HJ}$,

Tel: +82-2-958-5365

Fax: +82-2-958-5475

E-mail: almacore@kist.re.kr

Received March 23, 2015

Revised March 24, 2015

Accepted March 25, 2015
Dark field (DF) transmission electron microscopy image has become a popular characterization method for two-dimensional material, graphene, since it can visualize grain structure and multilayer islands, and further provide structural information such as crystal orientation relations, defects, etc. unlike other imaging tools. Here we present microstructure of graphene, particularly, using DF imaging. High-angle grain boundary formation wass observed in heat-treated chemical vapor deposition-grown graphene on the Si substrate using patch-quilted DF imaging processing, which is supposed to occur by strain around multilayer islands. Upon the crystal orientation between layers the multilayer islands were categorized into the oriented one and the twisted one, and their local structure were compared. In addition information from each diffraction spot in selected area diffraction pattern was summarized.

Key Words: Graphene, Transmission electron microscopy, Dark field image, Grain structure, Recrystallization

\section{INTRODUCTION}

The properties of materials could change considerably depending on dimensionality. A typical case such as graphite, it can be varied from zero-dimensional to three-dimensional materials. Graphene, two-dimensional (2D) material, a sheet form of graphite, is recently paid attention for their extraordinary physical properties and potential applications of graphene (Geim \& Novoselov, 2007; Castro Neto et al., 2009; Geim, 2009; Mas-Ballesté et al., 2011). The property of graphene is affected by their local structure such as impurity, defect, number of layers, stacking sequence etc., thus it is necessary to identify the quality of samples using several characterization tools (Hashimoto et al., 2004; Krasheninnikov et al., 2009; Zhang et al., 2009; Lui et al., 2011). Raman spectroscopy is often used because of easy sample preparation. D peak could represent defective level of graphene and intensity ratio of $2 \mathrm{D}$ to $\mathrm{G}$ exhibits number of graphene layers (Ferrari et al., 2006; Yan et al., 2011). However, such properties can be assured in only indirect way from Raman spectroscopy. To get further information on the local structure, high resolution transmission electron microscopy (TEM) is a great analysis tool since it provides real space images and crystallographic information simultaneously (Cockayne et al., 2011). Ryu et al. (2012) successfully imaged the atomic structure of tilted bilayer graphene by controlling defocus and $\mathrm{C}_{\mathrm{s}}$ correction value. Meanwhile, dark field TEM (DF-TEM) imaging is another technique to detect the local structure over large area rapidly. For polycrystalline graphene, hexagonal spots of graphene in selected area diffraction pattern (SADP) are

This work was supported by a grant of Korea Institute of Science and Technology (KIST) through 2 V04081.

@ This is an open-access article distributed under the terms of the Creative Commons Attribution Non-Commercial License (http://creativecommons.org/licenses/by-nc/3.0) which permits unrestricted noncommercial use, distribution, and reproduction in any medium, provided the original work is properly cited.

Copyrights @ 2015 by Korean Society of Microscopy 
rotated according to in-plane orientation of grains. Thus selection of one of the grain (or diffraction spot in SADP), that is DF imaging, visualizes the graphene grain structure while bright-field (BF) image shows no distinctive feature. In this article, we explore the local structure of graphene, specifically grain size or shape, structural sequence and defects using the DF-TEM imaging technique in polycrystalline and multilayer graphene.

\section{MATERIALS AND METHODS}

\section{Preparation of Graphene Samples}

Twenty-five micrometers thick Cu foils (99.8\%, No. 13382; Alfa Aesar, UK) were inserted into a $5 \mathrm{~cm}$ quartz tube, which was loaded inside a horizontal furnace. The tube was pumped to $600 \mathrm{mTorr}$, then heated up to $1,000^{\circ} \mathrm{C}$ with $50 \mathrm{sccm} \mathrm{Ar}$ and $20 \mathrm{sccm} \mathrm{H}_{2}$ mixture flow. It took around 50 minutes for both heating up and annealing. Subsequently, graphene growth was carried out for 30 minutes under the mixture flow of 30 sccm $\mathrm{CH}_{4}$ and $20 \mathrm{sccm} \mathrm{H}_{2}$. In order to induce slow cooling and prevent the tube from cracking, the furnace lid was kept closed until the temperature reached $800^{\circ} \mathrm{C}$. Then, the sample was rapidly cooled down by opening the lid of the furnace and blowing air. Polymethylmethacrylate (PMMA) is used to cover top of as-grown chemical vapor deposition (CVD)graphene on the $\mathrm{Cu}$ substrate by spin coating to support graphene after etching $\mathrm{Cu}$ the substrate.

\section{TEM Sample Preparation}

$\mathrm{Cu}$ substrate was etched by ammonium persulfate solution $\left(\left(\mathrm{NH}_{4}\right)_{2} \mathrm{~S}_{2} \mathrm{O}_{8}, 0.1 \mathrm{M}\right)$ during the night. After etching, the floating PMMA/graphene film rinsed with deionized water and was scooped by Au Quantifoil grid (Quantifoil Micro Tools GmbH, Germany) for TEM analysis. For removing the PMMA, a grid is soaked into warm acetone $\left(60^{\circ} \mathrm{C}\right)$ for 1 hour. Subsequently the sample was dried in air and baked at $100^{\circ} \mathrm{C}$ for 10 minutes to remove adsorbents on the graphene surface and prevent hydro carbon contamination during TEM work. TEM (Titan G1 80-300; FEI, USA) analysis is performed at an accelerating voltage of $80 \mathrm{kV}$ to prevent damage on the graphene sample.

\section{RESULTS AND DISCUSSION}

\section{Grain Structure}

There has been great effort to scale up the graphene sheet size for a variety of applications. It has been reported that large and high quality single layer graphene can be fabricated by CVD on polycrystalline $\mathrm{Cu}$ foils (Li et al., 2009; Bae et al., 2010; Cao et al., 2010). Since the large-scale sheet is not able to avoid polycrystalline with current technology (Li et al., 2010; Liu \& Yakobson, 2010; Yazyev \& Louie, 2010) and the grain boundary, which is to be defective region, is known to degrade the electrical (Li et al., 2010; Yazyev \& Louie, 2010) and mechanical properties (Grantab, 2010), controlling the grain size and the grain boundary properties is a critical issue to enhance the properties of graphene. However, limited thickness just with a few atomic layers prevents the conventional grain analysis method to be applied.

Duong et al. (2012) developed simple protocol to probe the graphene grain boundaries using optical microscopy, which enables the observation of large-area graphene directly without transfer from the $\mathrm{Cu}$ foil. Kim et al. (2012) tried to image the grain structure using polarized optical microscopy, visualizing the nematic liquid-crystal structure which is spincoated on graphene and epitaxially interacted with it. Fei et al. (2013) utilized scanning plasmon interferometry that detects plasmon interference acquired at the grain boundary. With leading edge analysis technique of $\mathrm{C}_{\mathrm{s}}$-corrected STEM in high spatial resolution, atomic structure of the defective area between grains, that is grain boundary, has been analyzed to have pentagons, heptagons and distorted hexagons instead of typical honeycomb hexagonal atomic arrangement (Huang et al., 2011). In order to get the grain map, Huang et al. (2011) developed DF patch-quilt method, which is great to recognize small-scale (under $\mu \mathrm{m}$ size) grains thanks to better spatial resolution compare to any other imaging tools. Even though it is not time-efficient way to map large area since each grain image should be obtained separately and quilted in one image through digital imaging process, it is frequently used due to further structural information simultaneously taken during the analysis process. Huang et al. (2011) used the technique in order to optimize the synthesis process to increase the grain size and Lee et al. (2013) measured polycrystalline graphene based on the grain map. Lee et al. (2015 accepted) also confirmed the original as-grown sample has large scale grains before heat treatment.

With the patch-quilt DF imaging method, here we compared the grain size and the shape in as-CVD graphene and annealed one (Fig. 1). The annealing was performed at $400^{\circ} \mathrm{C}$ under vacuum condition after a transfer of graphene on the Si substrate. To distinguish the grains with different crystallographic orientations, DF images were taken by placing an objective lens aperture (area radius about 130 $\mathrm{nm}$ of the sample) on the back focal plane to get selective diffracted beam from the diffraction spots. Second-order diffraction spots were selected to include one diffraction spot only as shown in inset diffraction pattern of Fig. 1. When you use first-order plane for DF imaging of polycrystalline graphene, it is hard to avoid to take closed placed spots together even in a smallest aperture. Graphene grain map is colored after collecting the same orientation grain images and subsequent gathering the grain image pieces. It was confirmed that high quality as-grown sample has large-scale polygonal 

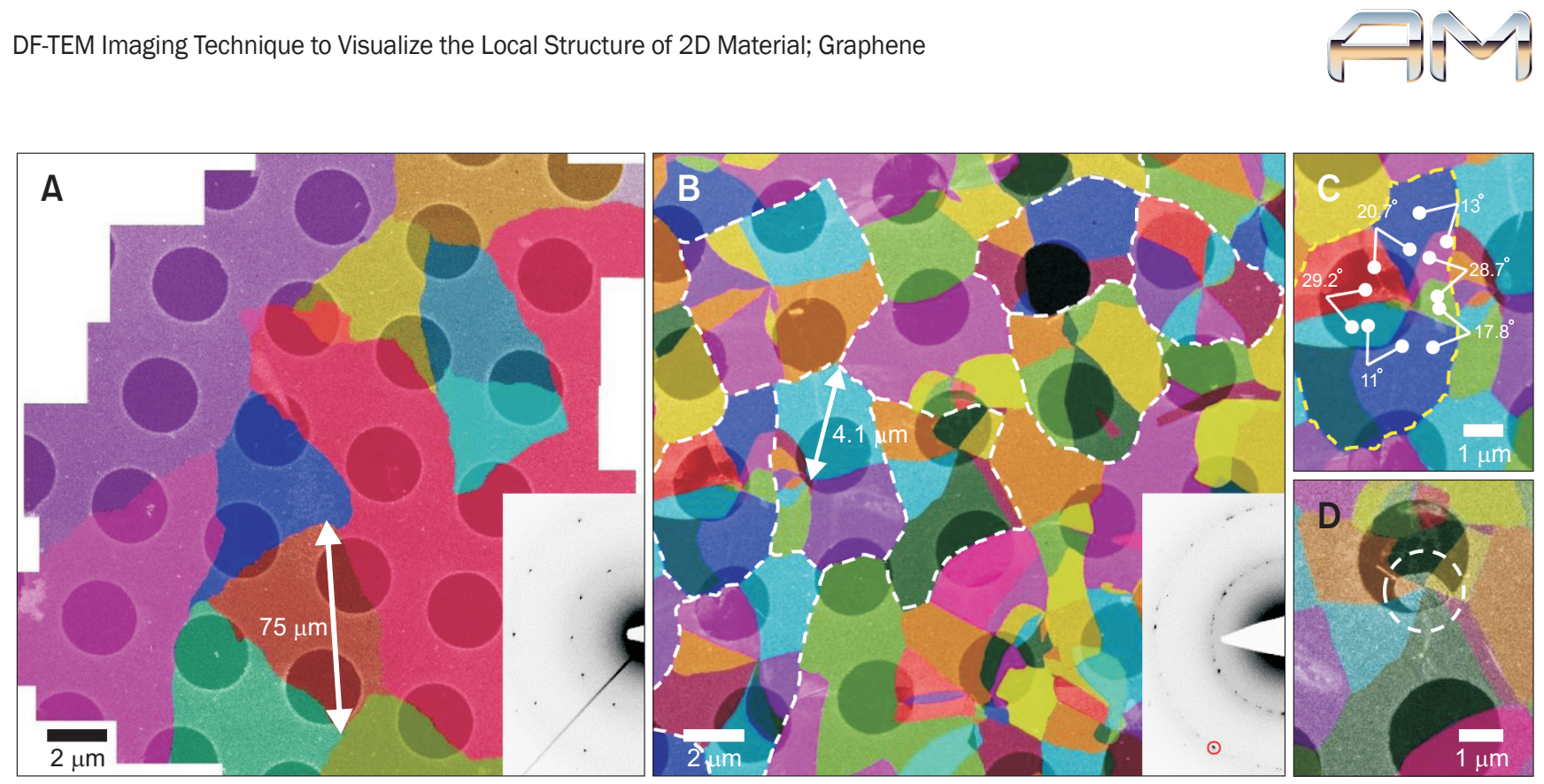

Fig. 1. Graphene grain maps, color-coded by crystal orientation, and corresponding selected area diffraction patterns (inset) for as-grown graphene (A) and annealed one at $400^{\circ} \mathrm{C}(\mathrm{B})$. Regions with radiant grains are marked with dotted white line. (C) Rotation angles between neighboring radiant grains are measured, indicating they have high-angle grain boundary. (D) Center area of radiant grains, where multilayer locates.

grains (Fig. 1A). Due to the large grain in as-grown sample, only two or three sets of diffraction patterns appear in SADP (inset), while annealed sample shows multiple spots (Fig. 1B) indicating it has smaller grains. In fact, the colored map shows that the grains are divided small pieces. Interestingly, grains grown with radial-shape are frequently observed as marked by dotted lines. The grain boundary angle is measured with the angle between the corresponding diffraction spots (Fig. 1C). The radial shaped grains are not just slightly (a few degree) rotated each other, but the rotation angle is large (mostly $10^{\circ} \sim 30^{\circ}$ ), which was confirmed by repeat measurement in many regions. This high-angle grain boundary indicates that recrystallization occurred even in low temperature of $400^{\circ} \mathrm{C}$. In most cases, multilayer islands are observed in the center of the radiant grains as shown in Fig. 1D. Thus it is expected that strain around multilayer during annealing is dominant factor to cause the recrystallization. In fact, Lee et al. (2015 accepted) presented that the estimated strain value generated during heat treatment on Si surface reach maximum at $400^{\circ} \mathrm{C}$. The Other minor nucleation site can be defects such as trapped $\mathrm{H}_{2} \mathrm{O}$ and $\mathrm{O}_{2}$ or impurities induced during transfer process to Si wafer.

\section{Multilayer Graphene}

It is known that graphene growth using CVD can produce multilayered-islands on the large area of single layer graphene. The multilayer graphene has been of scientific interest since they can be used to tune the physical properties of the graphene. The electronic, optical, and mechanical properties are dictated by the structure such as stacking order, defects, and interlayer spacing (Ohta et al., 2006; Lopes dos Santos et al., 2007; Avetisyan et al., 2010; Mele, 2010; Hicks et al., 2011; Luican et al., 2011; Shallcross et al., 2011; Suarez Morell et al., 2011). For example, bandgap opens with external perpendicular electric field in case of AB-stacked (Bernal) bilayer and ABC-stacked (rhombohedral) trilayer graphene, while ABA-stacked trilayer has no bandgap under same electric field (Castro et al., 2007; Zhang et al., 2009; Lui et al., 2011). In this regard, many researchers have put an effort to intentionally synthesize bi or multilayer graphene. Lee et al. (2010) synthesized a bi-layered Bernal graphene in wafer scale by controlling cooling rate in CVD process. Yan et al. (2011) reported that bilayer Bernal graphene can be synthesized by using two $\mathrm{Cu}$ substrates in a single CVD tube furnace. Shi et al. (2014 accepted) demonstrated that multi-layered graphene (up to 6 layers) can be produced by introducing a quasiclosed space to induce slow gas flow.

In order to examine qualities (like, homogeneity and continuity) of these multi-layered graphene, diverse characterization tools has been introduced. Raman spectroscopy has been used to indirectly identify a number of layers of graphene by intensity ratio between $2 \mathrm{D}$ to $\mathrm{G}$ peak and symmetry of 2D peak (Ferrari et al., 2006; Bhaviripudi et al., 2010; Lee et al., 2010; Yan et al., 2011). Atomic force microscopy has been used to directly visualize the multilayer graphene, though the instrumental offset error is necessary to be calibrated for better accuracy. Since graphite and graphene are both effective quencher reducing fluorescence intensity, fluorescence quenching microscopy (FQM) also can be used to image the graphene structure (Kim et al., 2009). Benefits of FQM are high-throughput and high-contrast imaging can be obtained on arbitrary substrate and even in solution (Kim et 
al., 2010).

Except above-mentioned methods, an optical microscopy offers an incomparably easier and better way to identify multilayered islands existing on the graphene. Even the thicknesses can be determined with high accuracy (Ni et al., 2007), when the graphene is prepared on top of Si wafers with a certain thickness of $\mathrm{SiO}_{2}$ and light wavelength (Abergel et al., 2007; Blakea et al., 2007). Fig. 2 shows a typical optical microscope image of graphene suspended on top of perforated Si wafer piece. The multi-layered graphene islands can be easily recognized by sharp contrast differences. As can be seen in Fig. 2, bi or trilayer islands are randomly distributed. The white lines are folded graphene, which might be caused by grain boundary of the Cu substrate.

The analysis tools mentioned above provide us the location of multilayer islands or number of layers, but no more structural

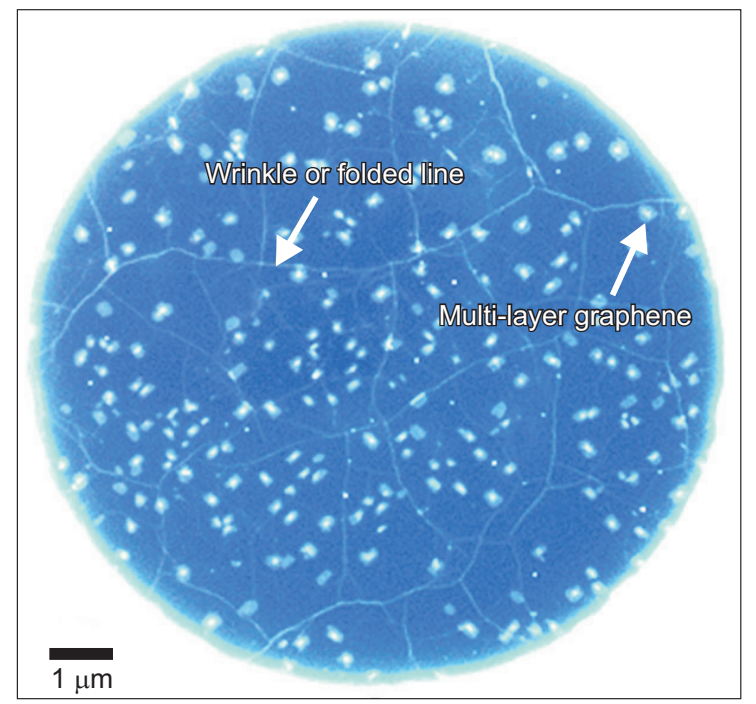

Fig. 2. Optical microscope image of graphene suspended on top of perforated Si wafer piece. Folded line and multilayer islands are visible. information. Further analysis for such as stacking sequence and defects as well as the number of layers or the density of islands requires DF-TEM technique (Brown et al., 2012; Alden et al., 2013). While no distinguished feature except carbon support film of grid appears in the BF image (Fig. $3 \mathrm{~A}$ ) due to weak signal from a mono or a few atomic layer of graphene, the DF image brightens area with specifically selected diffraction plane (Fig. 3B). It visualizes where the grain boundary is and how many layers the multilayer island has. It is trilayer and locates on the grain boundary covering two neighboring grains.

It has been proved that the multilayer can grow having stacking order with lower layer (called oriented layer), which is preferential energetically, but not in all cases. Brown et al. (2012) evaluated the statistical fraction of oriented bilayer area (70\%) in a graphene sheet grown by CVD method. The other

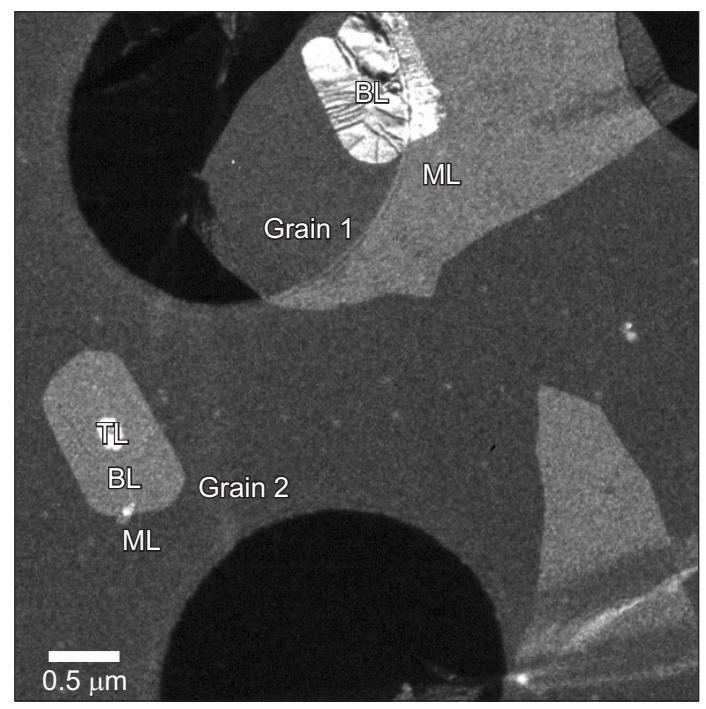

Fig. 4. Dark field image showing oriented trilayer (TL, lower left) in 'grain 1 ' and twisted bilayer (BL, upper right) in 'grain 2.' ML, monolayer.
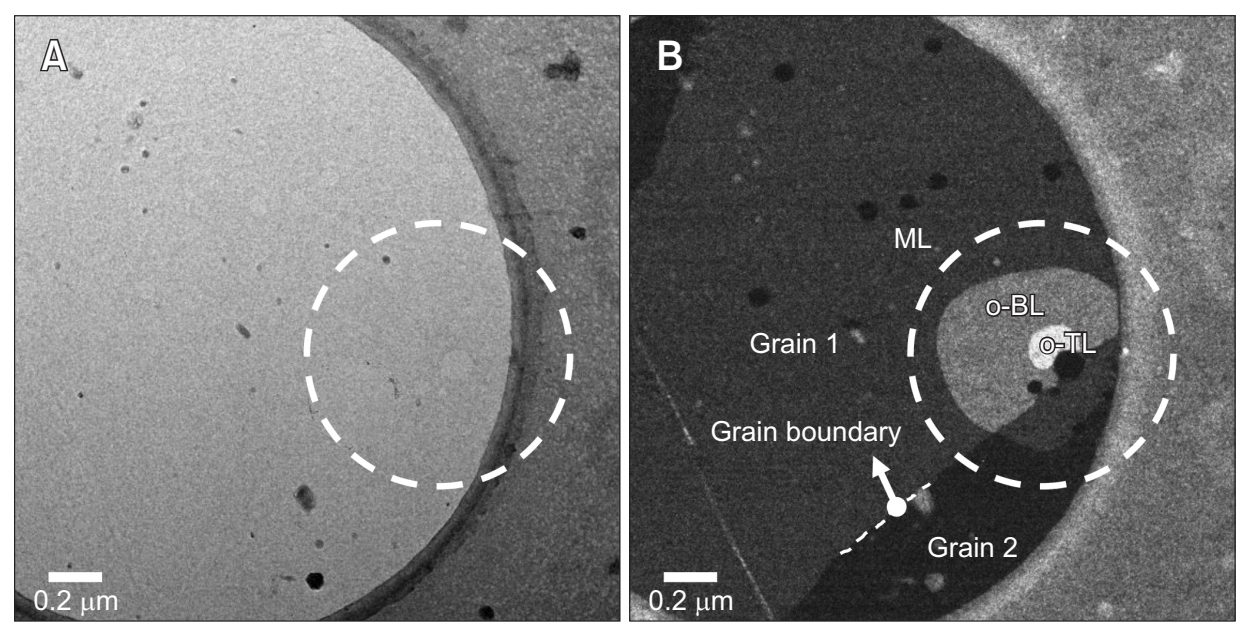

Fig. 3. Bright-field (A) and dark field (B) images of graphene placed on quantifoil grid. 'Grain 1' looks relatively bright, while 'grain 2' is dark. Multilayer (indicated by a white circles) placed across the grain boundary. Bilayer (BL) and trilayer (TL) in 'grain 1 ' is oriented layers with monolayer (ML). 
$30 \%$ had twisted hexagonal symmetry between upper and lower layer. In the article below, stacking sequence and defects in oriented or twisted multilayer graphene are reviewed.

\section{Oriented Multilayer Graphene}

In many cases, oriented multilayer islands has faceted polygonal edges (mostly distorted hexagonal shape) like the lower left one in Fig. 4 and the third layer having similar shape with the second one locates in the center of the second layer while the non-oriented one are irregular in shape and location (see upper right multilayer island in Fig. 4).

For the oriented multilayer, the DF imaging can identify the
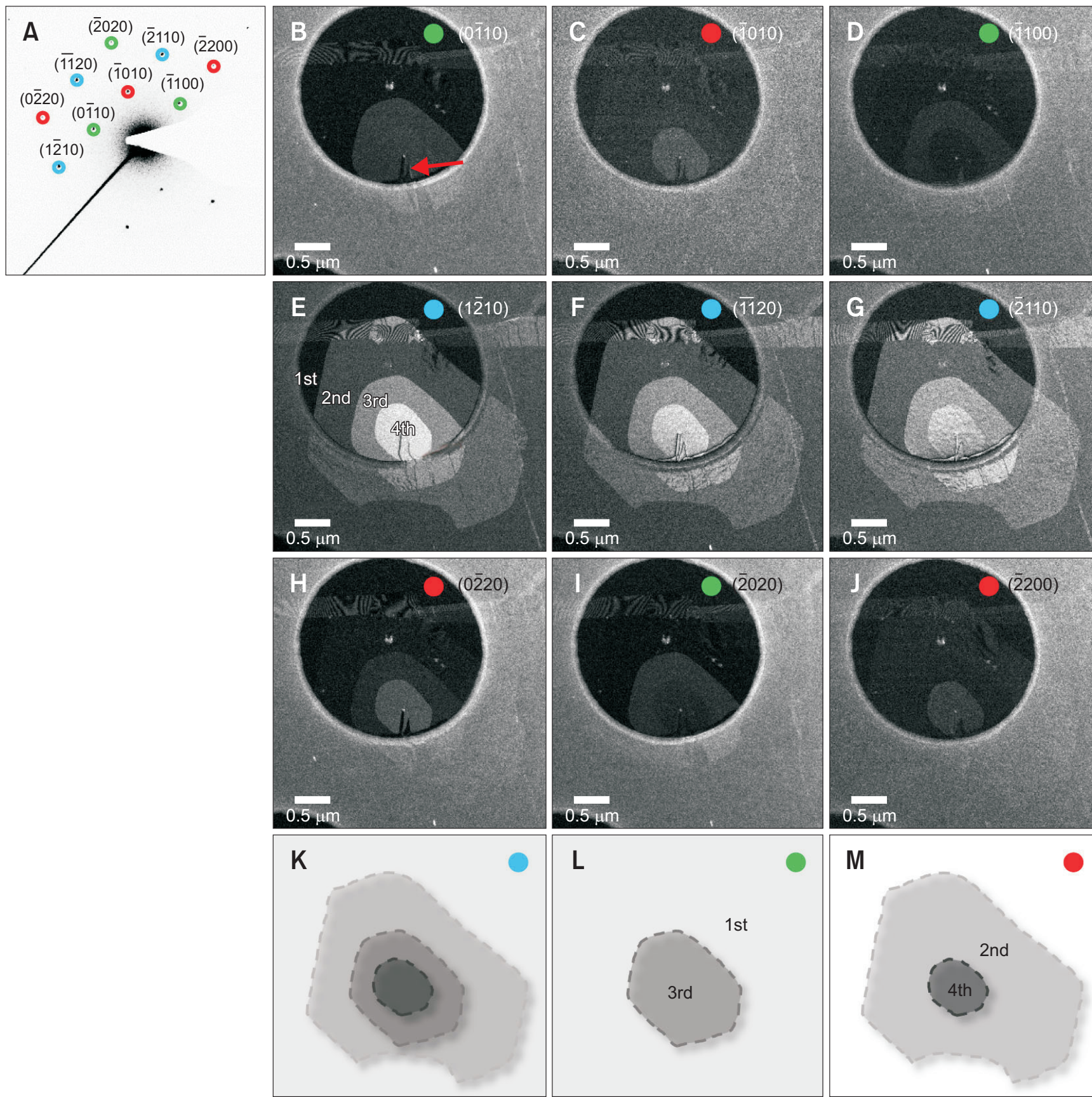

Fig. 5. Oriented tetralayer island. (A) Selected area diffraction pattern (SADP) of typical hexagonal symmetry without any extra spots; corresponding dark field images obtained from the first-order (B-D), the second-order (E-G) and the third-order (H-J) diffraction spots in SADP. (K-M) Schematics of highlighted layers in dark field images depending on selected diffraction spots. Diffraction spot highlighting odd layers like are marked by green circle (L), even layers like are red $(\mathrm{M})$ and all layers like are blue $(\mathrm{K})$ in $(\mathrm{A})$. 
number of graphene layers and stacking sequence (Ping \& Fuhrer, 2012) . Here we applied the analysis method for a tetralayer in Fig. 5. Each diffraction spot provides different DF images. One of the first-order diffraction spot, indexed as $(0 \overline{1} 10)$, visualizes the 1st and 3rd (odd-numbered) layer, while the next one $60^{\circ}$ rotated highlights 2 nd and 4 th (evennumbered) layer. Further $60^{\circ}$ rotated spot (1100) shows the 1st and 3rd again, which indicates that each layer has 3-fold symmetry. Schematics in Fig. 5I and M represent the highlighted layers in Fig. 5C, E, and D, respectively. The second-order spots with higher intensities in SADP provide enough contrast to all layers, which is sufficient to identify layer number. The contrast increase from monolayer to tetralayer region is linear. Here, it can be noticed that secondorder diffraction is useful to visualize multiple layers clearly as well as grain mapping. The third-order spot $(0 \overline{2} 20)$ aligned with $(0 \overline{1} 10)$ which makes odd-numbered layers visible let even-numbered layers brighter. The next third-order spot $((\overline{2} 020))$ activates odd-numbered layers, while spot rotated $60^{\circ}$ more $((\overline{2} 200))$ enables even-numbered layers visible again, which shows the third-order spots reflect the 3-fold symmetry like the first-order ones do.

Crystalline graphite is a strongly anisotropic layered solid consisting of a periodic stacking of graphene layers. One of the stacking sequences is Bernal-stacking, which has one corner of the hexagons of the second sheet located above the center of the hexagons of the lower sheet. This is known to be well-stacked arrangement and be the lowest energy. In a Bernal-stacked trilayer (ABA), the third would have the same relationship to the middle layer and its atoms would appear at the position coincident with the carbons in the lower sheet looking directly down on the stack. There is also other arrangement, a rhombohedral-stacked (ABC) trilayer, in which the upper sheet is shifted by the distance of an atom, so that the upper sheet is related to the lower sheet in the Bernal arrangement. Ping \& Fuhrer (2012) proposed to distinguish the $\mathrm{ABC}$ stack from $\mathrm{ABA}$ with the lower intensity in the first-order DF image, since the contrast intensity grows monotonically with layer number for the Bernal-stacked region, but is low and non-monotonic for rhombohedrally stacked region. The tetralayer in Fig. 5 is identified as Bernal stacked (ABAB) layer. We used $I_{1} / I_{2}$ ratio method, which is explained in detail in refs (Ping \& Fuhrer, 2012). The ratio of the first-order diffraction spot over the second-order one is 0.32 for Fig. $5 \mathrm{~A}$, which matches close to the theoretical value of 0.28 for the layer number of 4 . The corresponding structure model is 2, 2, which means there are two layers of A stack position and two layers of $\mathrm{B}$, that is Bernal stacking. Additionally, it can be double checked by tilting the sample and measuring the intensity change of diffraction spots in SADP (Brown et al., 2012; supporting information).

It is noticeable a few long triangle dark regions in the lower part of trilayer and tetralayer in Fig. 5B (marked by an arrow) and the second-order DF images (Fig. 5E-G) show dark lines. According to Ping \& Fuhrer's report (2012), the dark region is expected to have different stacking order, rhombohedral stacking (ABCA) in this case. Different stacking order domain here is not expected to be intrinsic defect, rather formed by mechanical stress due to bending event at the edge of carbon film of grid since boundary lines are observed only around the edge of the carbon film edge (Fig. 5E-G). Brown et al. (2012) also observed two distinguished parallel contrast
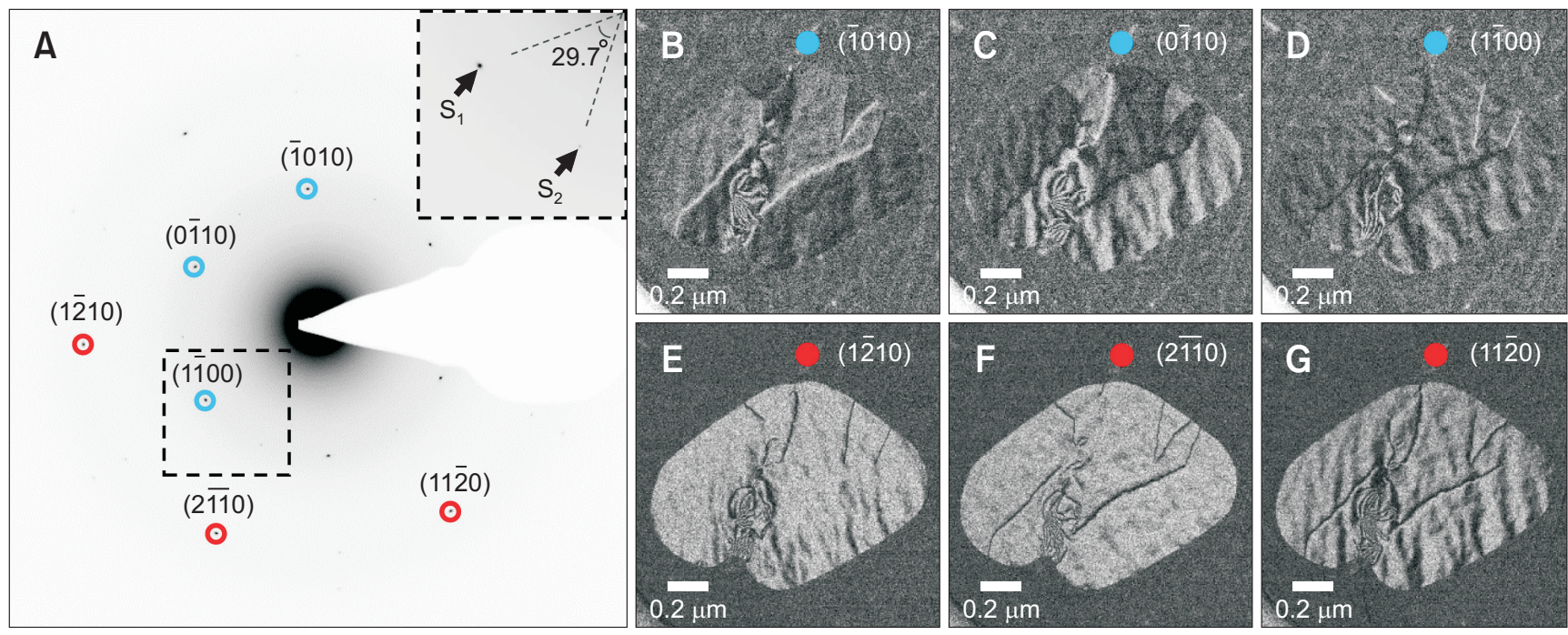

Fig. 6. (A) Selected area diffraction pattern obtained from bilayer region. The dotted square is magnified in inset. The rotation angle between $S_{1}$ and $S_{2}$ is 29.7 ${ }^{\circ}$. Dark field images are taken from the first-order diffraction spots (marked as blue) (B-D) the second-order diffraction spots (marked as red) (E-G). Visualize defective domains with different contrast (B-D) and shows the boundaries between them (E-G). 
in a layer and confirmed it as a twin defect with a different stacking configuration (AC) by performing a tilt-dependent SADP analysis.

This systematic approach to DF images provides a simple tip which spot you need to use upon the interest. The number of layers can be easily identified when the secondorder diffraction spot is selected. The boundary of stacking sequence domain or line defect also can be recognized. The first- or third-order spots visualize A and B layers by turns and area with different stacking order is distinguished with different contrast intensity.

\section{Twisted Multilayer Graphene}

Now we show a typical twisted multilayer graphene in Fig. 6. SADP obtained from bilayer region shows two different hexagonal sets (Fig. 6A). The dotted square is magnified in inset, Diffraction spot $S_{1}$ comes from monolayer, while $S_{2}$ with less diffraction signal is supposed to come from the second layer. The twisted angle between layers can be measured from the relative rotation angle between neighboring diffraction spots.

The second layer is rotated $29.7^{\circ}$ from the first one, which locate the first-order diffraction spot very close to the halfdistance position of ( $2 \overline{11} 0$ ) by coincidence. The small stacking defect such as a rotation is known to have a profound effect on the electronic structure according to theoretical calculations (Lopes dos Santos et al., 2007; Mele, 2010). The properties deviates from the one of Bernal stacked bilayer that opens bandgap. In general, the twisted multilayer shows more defective features than oriented one. DF images taken from the first-order diffraction spots (Fig. 5B-D) show the second layer is divided into several domains which is supposed to have different orientation and their domain boundaries are visible in second-order DF images (Fig. 5E-G).

Strain contour were observed all over the bilayer region, showing that the second layer is strained due to mismatch with the first layer and divided domains in the second layer. When the rotation angle is small enough, producing close diffraction spots in SADP, for the aperture to take them both for the DF imaging, Moiré fringes are observed. More Moiré pattern images experimentally observed can be found in Brown et al. (2012), even in atomic resolution scale (Robertson et al., 2011; Ryu et al., 2012). The strain on the layer was analyzed with Moiré fringes, curve pattern implies that pre- strain and shear-strain elements are both present (Brown et al., 2012).

\section{CONCLUSIONS}

In this paper, we demonstrated DF-TEM technique to visualize and investigate the structure of graphene; 1 ) the grain size and shape, 2) number of multilayer, crystal orientation between layers, stacking sequence and defects in multilayer graphene.

The grain structure of CVD-grown graphene was explored using the patch-quilt DF imaging method. As a result, it was found that annealing transferred graphene on the Si wafer after initial growth causes recrystallization. Radiant grains have high-angle grain boundary the nucleation is expected to occur by strain around multilayer islands.

Upon the interest, different diffraction spots should be selected for DF imaging. The second-order diffraction spot brightens all layers with enough contrast, which let us identify the number of layers. It also shows domain boundaries, of course different lines depending on selected crystal plane. Diffraction spots on the first or third-order plane highlight odd or even layers by $60^{\circ}$ turns. In addition, domains having different stacking sequence are distinguished due to different contrast in DF image.

The multilayer islands are categorized into oriented one and twisted one depending on the crystal orientation between layers. When the number of layer is $>3$, it is easily distinguished with shape and relative position of upper layer to lower one. Twisted bilayer can be identified by extra set of hexagonal shape diffraction spots in SADP.

Compared to other imaging techniques, big advantage of DF imaging combined with SADP is to provide crystal structural information in a relatively high resolution. This is not limited to only graphene, but also can be applied widely to all twodimensional materials such as hexagonal boron nitride and semiconducting transition metal dichalcogenides $\mathrm{MX}_{2}$ ( $\mathrm{M}=\mathrm{Mo}, \mathrm{W} ; \mathrm{X}=\mathrm{S}, \mathrm{Se}$ ) (Kim et al., 2013; van der Zande et al., 2013).

\section{CONFLICT OF INTEREST}

No potential conflict of interest relevant to this article was reported.

\section{REFERENCES}

Abergel D S L, Russell A, and Fal'ko V I (2007) Visibility of a graphene flake on a dielectric substrate. Appl. Phys. Lett. 91, 063125.

Alden J S, Tsen A W, Huang P Y, Hovden R, Brown L, Park J, Muller D A, and McEuen P L (2013) Strain solitons and topological defects in bilayer graphene. Proc. Natl. Acad. Sci. 110, 11256-11260.

Avetisyan A A, Partoens B, and Peeters F M (2010) Stacking order 
dependent electric field tuning of the band gap in graphene multilayers. Phys. Rev. B 81, 115432.

Bae S, Kim H, Lee Y, Xu X, Park J S, Zheng Y, Balakrishnan J, Lei T, Kim H R, Song Y I, Kim Y J, Kim K S, Özyilmaz B, Ahn J H, Hong B H, and lijima S (2010) Roll-to-roll production of 30-inch graphene films for transparent electrodes. Nat. Nanotechnol. 5, 574-578.

Bhaviripudi S, Jia X, Dresselhaus M S, and Kong J (2010) Role of kinetic factors in chemical vapor deposition synthesis of uniform large area graphene using copper catalyst. Nano Lett. 10, 4128-4133.

Blakea P, Hill E W, Castro Neto A H, Novoselov K S, Jiang D, Yang R, Booth T J, and Geim A K (2007) Making graphene visible. Appl. Phys. Lett. 91, 063124

Brown L, Hovden R, Huang P, Wojcik M, Muller D A, and Park J (2012) Twinning and twisting of tri- and bilayer graphene. Nano Lett. $\mathbf{1 2}$, 1609-1615.

Cao H, Yu Q, Jauregui L A, Tian J, Wu W, Liu Z, Jalilian R, Benjamin D K, Jiang Z, Bao J, Pei S S, and Chen Y P (2010) Electronic transport in chemical vapor deposited graphene synthesized on $\mathrm{Cu}$ : quantum hall effect and weak localization. Appl. Phys. Lett. 96, 122106.

Castro E, Novoselov K, Morozov S, Peres N, dos Santos J, Nilsson J, Guinea F, Geim A, and Neto A (2007) Biased bilayer graphene: semiconductor with a gap tunable by the electric field effect. Phys. Rev. Lett. 99, 216802

Castro Neto A H, Guinea F, Peres N M R, Novoselov K S, and Geim A K (2009) The electronic properties of graphene. Rev. Mod. Phys. $\mathbf{8 1}$ 109-162.

Cockayne E, Rutter G M, Guisinger N P, Crain J N, First P N, and Stroscio J A (2011) Grain boundary loops in graphene. Phys. Rev. B 83, 195425.

Duong D L, Han G H, Lee S M, Gunes F, Kim E S, Kim S T, Kim H, Ta Q H, So K P, Yoon S J, Chae S J, Jo Y W, Park M H, Chae S H, Lim S C, Choi J Y, and Lee Y H (2012) Probing graphene grain boundaries with optical microscopy. Nature 490, 235-239.

Fei Z, Rodin A S, Gannett W, Dai S, Regan W, Wagner M, Liu M K, McLeod A S, Dominguez G, Thiemens M, Castro Neto A H, Keilmann F, Zettl A, Hillenbrand R, Fogler M M, and Basov D N (2013) Electronic and plasmonic phenomena at graphene grain boundaries. Nat. Nanotechnol. 8, 821-825.

Ferrari A C, Meyer J C, Scardaci V, Casiraghi C, Lazzeri M, Mauri F, Piscanec S, Jiang D, Novoselov K S, Roth S, and Geim A K (2006) Raman spectrum of graphene and graphene layers. Phys. Rev. Lett. 97, 187401.

Geim A K (2009) Graphene: status and prospects. Science 324, 15301534.

Geim A K and Novoselov K S (2007) The rise of graphene. Nat. Mater. 6 183-191.

Grantab R, Shenoy V B, and Ruoff R S (2010) Anomalous strength characteristics of tilt grain boundaries in graphene. Science $\mathbf{3 3 0}$, 946-948.

Hashimoto A, Suenaga K, Gloter A, Urita K, and lijima S (2004) Direct evidence for atomic defects in graphene layers. Nature 430, 870-873.

Hicks J, Sprinkle M, Shepperd K, Wang F, Tejeda A, Taleb-Ibrahimi A, Bertran F, Le Fèvre P, de Heer W A, Berger C, and Conrad E H (2011) Symmetry breaking in commensurate graphene rotational stacking: a comparison of theory and experiment. Phys. Rev. B 83, 205403.

Huang P Y, Ruiz-Vargas C S, van der Zande A M, Whitney W S, Levendorf M P, Kevek J W, Garg S, Alden J S, Hustedt C J, Zhu Y, Park J, McEuen P L, and Muller D A (2011) Grains and grain boundaries in single- layer graphene atomic patchwork quilts. Nature 469, 389-393.

Kim C J, Brown L, Graham M W, Hovden R, Havener R W, McEuen P L, Muller D A, and Park J (2013) Stacking order dependent second harmonic generation and topological defect in h-BN bilayers. Nano Lett. 13, 5660-5665.

Kim D W, Kim Y H, Jeong H S, and Jung H T (2012) Direct visualization of large-area graphene domains and boundaries by optical birefringency. Nat. Nanotechnol. 7, 29-34.

Kim J, Cote L J, Kim F, and Huang J (2009) Visualizing graphene based sheets by fluorescence quenching microscopy. J. Am. Chem. Soc. 132, 260-267.

Kim J, Kim F, and Huang J (2010) Seeing graphite-based sheets. Materials Today 13, 28-38.

Krasheninnikov A V, Lehtinen P O, Foster A S, Pyykkö P, and Nieminen R M (2009) Embedding transition-metal atoms in graphene: structure, bonding, and magnetism. Phys. Rev. Lett. 102, 126807.

Lee G H, Cooper R C, An S J, Lee S, van der Zande A, Petrone N, Hammerberg A G, Lee C, Crawford B, Oliver W, Kysar J W, and Hone J (2013) High-strength chemical-vapor-deposited graphene and grain boundaries. Science 340, 1073-1076.

Lee S, Lee K, and Zhong Z (2010) Wafer scale homogeneous bilayer graphene films by chemical vapor deposition. Nano Lett. 10, 47024707.

Lee S M, Kim S M, Na M Y, Chang H J, Kim K S, Yu H, Lee H J, and Kim J $\mathrm{H}$ (2005 accepted) Materialization of strained CVD-graphene using thermal mismatch. Accepted for publication in Nano Res.

Li X, Cai W, An J, Kim S, Nah J, Yang D, Piner R, Velamakanni A, Jung I, Tutuc E, Banerjee S K, Colombo L, and Ruoff R S (2009) Large-area synthesis of high-quality and uniform graphene films on copper foils. Science 324, 1312-1314.

Li X, Magnuson C W, Venugopal A, An J, Suk J W, Han B, Borysiak M, Cai W, Velamakanni A, Zhu Y, Fu L, Vogel E M, Voelkl E, Colombo L, and Ruoff R S (2010) Graphene films with large domain size by a twostep chemical vapor deposition process. Nano Lett. 10, 4328-4334.

Liu Y and Yakobson B I (2010) Cones, pringles, and grain boundary landscapes in graphene topology. Nano Lett. 10, 2178-2183.

Lopes dos Santos J M B, Peres N M R, and Castro Neto A H (2007) Graphene bilayer with a twist: electronic structure. Phys. Rev. Lett. 99, 256802

Lui C H, Li Z, Mak K F, Cappelluti E, and Heinz T F (2011) Observation of an electrically tunable band gap in trilayer graphene. Nat. Phys. 7, 944-947.

Luican A, Li G, Reina A, Kong J, Nair R, Novoselov K, Geim A, and Andrei E (2011) Single-layer behavior and its breakdown in twisted graphene layers. Phys. Rev. Lett. 106, 126802.

Mas-Ballesté R, Gómez-Navarro C, Gómez-Herrero J, and Zamora F (2011) 2D materials: to graphene and beyond. Nanoscale 3, 20-30.

Mele E J (2010) Commensuration and interlayer coherence in twisted bilayer graphene. Phys. Rev. B 81, 161405R.

Ni Z H, Wang H M, Kasim J, Fan H M, Yu T, Wu Y H, Feng Y P, and Shen Z X (2007) Graphene thickness determination using reflection and contrast spectroscopy. Nano Lett. 7, 2758-2763.

Ohta T, Bostwick A, Seyller, T, Horn K, and Rotenberg E (2006) Controlling the electronic structure of bilayer graphene. Science 313, 951-954.

Ping J and Fuhrer M S (2012) Layer number and stacking sequence imaging of few-layer graphene by transmission electron microscopy. Nano Lett. 12, 4635-4641. 
Robertson A W, Bachmatiuk A, Wu Y A, Schäffel F, Rellinghaus B, Büchner B, Rümmeli M H, and Warner J H (2011) Atomic structure of interconnected few-layer graphene domains. ACS Nano 5, 66106618.

Ryu G H, Park H J, Kim N Y, and Lee Z (2012) Atomic resolution imaging of rotated bilayer graphene sheets using a low kV aberrationcorrected transmission electron microscope. Appl. Microsc. 42, 218222.

Shallcross S, Sharma S, Landgraf W, and Pankratov O (2011) Electronic structure of graphene twist stacks. Phys. Rev. B 83, 054502.

Shi Y, Wang D, Zhang J, Zhang P, Shi X, and Hao Yue (2014 accepted) Synthesis of Multilayer graphene films on copper by modified chemical vapor deposition. Accepted for publication in Mater. Manuf. Process.

Suaŕez Morell E, Vargas P, Chico L, and Brey L (2011) Charge redistribution and interlayer coupling in twisted bilayer graphene under electric fields. Phys. Rev. B 84, 195421.

van der Zande A M, Huang P Y, Chenet D A, Berkelbach T C, You Y M, Lee G H, Heinz T F, Reichman D R, Muller D A, and Hone J C (2013) Grains and grain boundaries in highly crystalline monolayer molybdenum disulphide. Nat. Mater. 12, 554-561.

Yan K, Peng H, Zhou Y, Li H, and Liu Z (2011) Formation of bilayer bernal graphene: layer-by-layer epitaxy via chemical vapor deposition. Nano Lett. 11, 1106-1110.

Yazyev O V and Louie S G (2010) Electronic transport in polycrystalline graphene. Nat. Mater. 9, 806-809.

Zhang Y, Tang T T, Girit C, Hao Z, Martin M C, Zettl A, Crommie M F, Shen Y R, and Wang, F (2009) Direct observation of a widely tunable bandgap in bilayer graphene. Nature $459,820-823$. 